\title{
THE NUMBER OF FINITE TOPOLOGIES ${ }^{1}$
}

\author{
D. KLEITMAN ${ }^{2}$ AND B. ROTHSCHILD
}

Abstract. The logarithm (base 2) of the number of distinct topologies on a set of $n$ elements is shown to be asymptotic to $n^{2} / 4$ as $n$ goes to infinity.

Let $X$ be a set with $n$ elements, and let $T(X)$ be the set of all topologies that can be defined on $X$. Then we set $T_{n}=|T(X)|$. The number $T_{n}$ has been determined for certain small values of $n$ [4]. $T_{n}$ has been estimated by several authors [2], [3], [8]. We present here an asymptotic estimate for the logarithm of $T_{n}$. We do this by considering an equivalent problem. Namely, if $P(X)$ denotes the set of all partial orders that can be defined on a set $X$ with $n$ elements, and if we set $P_{n}=|P(X)|$, then we estimate $P_{n}$. The sizes of certain special subsets of $P(X)$ have been determined, and thus provide lower bounds for $P_{n}$ [6], [7].

We begin by presenting several enumeration problems which are equivalent to one another in a certain sense (see Lemma 1 below). Let $T_{n}$ and $P_{n}$ be defined as above. Similarly, for $X$ a set with $n$ elements, let $T_{0}(X)$ be the set of all $T_{0}$-topologies that can be defined on $X$ (i.e., if $a, b$ are two elements of $X$, then there is some open set containing one but not both of them [5]), and let $O(X)$ be the set of all preorders that can be defined on $X$ (i.e., reflexive and transitive, but not necessarily antisymmetric). Then set $T_{n, 0}=\left|T_{0}(X)\right|$, and $O_{n}=|O(X)|$. Let $T_{n}^{\prime}, P_{n}^{\prime}, T_{n, 0}^{\prime}, O_{n}^{\prime}$ denote the numbers of isomorphism classes in $T(X), P(X), T_{0}(X), O(X)$ respectively.

From [2] we know that

$$
T_{n} \geqq 2^{n^{2} / 4}
$$

(Alternatively, we have $P_{n} \geqq 2^{n^{2} / 4}$, which we observe trivially after the introduction of "diagrams" below.)

Lemma 1. $T_{n}=O_{n}, T_{n, 0}=P_{n}, T_{n}^{\prime}=O_{n}^{\prime}, T_{n, 0}^{\prime}=P_{n}^{\prime}$. All eight of these quantities have logarithms which are asymptotically equal as $n$ tends to infinity.

Received by the editors, August 27, 1969.

AMS Subject Classifications. Primary 0565.

Key Words and Phrases. Partial order, finite set, asymptotic enumeration.

1 Research sponsored in part by the Air Force Office of Scientific Research, Office of Aerospace Research, under contract No. F44620-67-6-0029.

2 Supported in part by NSF Grant GP-13778. 
Note. In the statement of Lemma 1 and everywhere else in this paper all logarithms are of base 2 .

Proof. The four equalities are well known [1], [2]. For the second part of the lemma, in view of (1), it need only be shown that $\log \left(T_{n} / T_{n}^{\prime}\right) \cdot \log \left(T_{n, 0} / T_{n, 0}^{\prime}\right)$ and $\log \left(O_{n} / P_{n}\right)$ are all $o\left(n^{2}\right)$. Since $T_{n} / T_{n}$, and $T_{n, 0} / T_{n, 0}$, are at most $n !$, and $\log n !$ is $o\left(n^{2}\right)$, it remains only to show that $\log \left(O_{n} / P_{n}\right)=o\left(n^{2}\right)$. To obtain a preorder on a set $X$ with $n$ elements merely partition $X$ into subsets (in at most $n ! 2^{n-1}$ ways), and then partially order the subsets (in at most $P_{n}$ ways) [2]. But $\log \left(n ! 2^{n-1}\right)=o\left(n^{2}\right)$. This completes the proof.

There is associated with each partial order a unique Hasse diagram, or just diagram (basis graph in [9]). It consists of a graph having as vertices the elements of the set, and for each pair $a, b$ of these vertices an edge directed from $a$ to $b$ iff $a$ covers $b$, that is, $a>b$, and for no $c$ is $a>c>b$. Thus, a diagram will be a directed graph which avoids two types of configurations: a polygon with its edges directed cyclically, or a polygon with all but one edge directed cyclically. For example, a bipartite graph with its edges all directed from just one of its two parts to the other is such a diagram. There are clearly at least $2^{n^{2} / 4}$ of these, and thus $P_{n}>2^{n^{2} / 4}$. Estimating the number of diagrams is the same as estimating the number of partial orders, but we find the former concept more convenient.

Theorem 1. For some constant $C, \log P_{n} \leqq n^{2} / 4+C n^{3 / 2} \log n$ for all $n$.

The proof of this theorem will be by induction on $n$. We show that all diagrams on $n+1$ vertices can be obtained from those with $n$ or fewer vertices by adjoining vertices and edges in certain specified ways. The induction is accomplished by observing that if we start with smaller diagrams and obtain all possible diagrams on $n+1$ vertices by these methods, then the inequality of Theorem 1 is preserved. Each of the three following lemmas concerns itself with one of the special methods and will be used to show that the inequality is preserved in the induction step. Together they include all possible diagrams on $n+1$ vertices.

For any set $V$ let $A(V)$ denote the set of diagrams that can be defined with $V$ as vertices such that some vertex is adjacent to (i.e., covering or covered by) at most $(|V|-1) / 64$ of the other vertices. When $|V|=k$, set $A_{k}=|A(V)|$.

LEMmA 2. $\log \left(A_{n+1} / P_{n}\right)<n / 5$ for $n$ sufficiently large.

Proof. Let $|V|=n+1$. We can obtain any member of $A(V)$ by starting with some diagram on $n$ of the vertices of $V$ and adjoining 
the $(n+1)$ st vertex with at most $n / 64$ vertices covering it, and at most $n / 64$ covered by it. There are $\left(\begin{array}{c}n+1 \\ n\end{array}\right) P_{n}$ ways to pick a diagram on just $n$ of the vertices. The remaining vertex can be connected to some number, up to $n / 64$, of vertices covering it. There are

$$
\sum_{i=0}^{[n / 64]}\left(\begin{array}{l}
n \\
i
\end{array}\right) \leqq \frac{1}{64} n\left(\begin{array}{c}
N \\
{[n / 64]}
\end{array}\right)
$$

ways to choose these. The number of vertices which it covers can then be chosen in even fewer ways. Thus, we have

$$
\log \left(\frac{A_{n+1}}{P_{n}}\right) \leqq \log (n+1)+2 \log \frac{n}{64}+2 \log \left(\begin{array}{c}
n \\
{[n / 64]}
\end{array}\right) .
$$

Using Stirling's formula, we have, for $n$ sufficiently large,

$$
\begin{aligned}
\log \left(\frac{A_{n+1}}{P_{n}}\right) \leqq & \log (n+1)+2 \log \frac{n}{64} \\
& -\log \left(2 \pi n \frac{1}{64} \frac{63}{64}\right)-\frac{2 n}{64} \log \left(\frac{1}{64}\right)-\frac{2 n 63}{64} \log \frac{63}{64} .
\end{aligned}
$$

Clearly, then, for $n$ sufficiently large

$$
\log \left(\frac{A_{n+1}}{P_{n}}\right) \leqq \frac{21}{64} n+2 n \log \frac{64}{63}<\frac{n}{5} .
$$

This proves the lemma.

Definition. A vertex of a diagram is minimal if it covers no other vertex.

Definition. Let $Q$ be a set of vertices in a diagram. Then $C(Q)$ denotes the set of vertices in the diagram which are adjacent to (i.e., covering or covered by) any of the vertices in $Q$.

Let $V$ be a set, and let $|V|=k+1$. Denote by $B(V)$ the set of diagrams that can be defined with $V$ as vertices and with a minimal vertex covered by a set $Q$ of size $\left[k^{1 / 2}\right]$, with $C(Q)$ containing at least $k / 2$ vertices. Let $B_{k+1}=|B(V)|$.

Lemma 3. $\log \left(B_{n+1} / P_{n}\right)<n / 2+n^{1 / 2} \log n$ for sufficiently large $n$.

Proof. First we observe that no diagram can contain a triangle, since no matter how the edges of the triangle are directed one of the forbidden configurations occurs. Let $|V|=n+1$. Any diagram in $B(V)$ can be obtained by starting with a diagram on just $n$ of the vertices of $V$ (there are $\left(\begin{array}{c}n+1 \\ n\end{array}\right) P_{n}$ choices for the diagram on $n$ of the 
vertices), and adjoining the $(n+1)$ st vertex $v$ as a minimal vertex covered by a set $Q$ of other vertices with $|Q|=\left[n^{1 / 2}\right],|C(Q)| \geqq n / 2$. There are at most $\left({ }_{\left[n^{n / 2}\right]}^{n}\right)$ ways to choose $Q$. (Some of the $\left.{ }_{\left[n^{n / 2}\right]}^{n}\right)$ choices for $Q$ either will violate the condition on $C(Q)$ or will produce one of the forbidden configurations.) Finally, once $Q$ is selected satisfying $|C(Q)| \geqq n / 2$, then there are at most $n / 2-\left[n^{1 / 2}\right]$ remaining vertices to which $v$ might be adjacent. ( $v$ cannot be adjacent to any vertex in $C(Q)$, otherwise there would be a triangle.) Thus there are at most $2^{n / 2-\left[n^{1 / 2}\right]}$ ways to connect $v$ to the remaining vertices not in $Q$ or $C(Q)$. We have

$$
\begin{aligned}
\log \left(\frac{B_{n+1}}{P_{n}}\right) & \leqq \log (n+1)+\log \left(\begin{array}{c}
n \\
{\left[n^{1 / 2}\right]}
\end{array}\right)+\frac{n}{2}-\left[n^{1 / 2}\right] \\
& <\log \left(\begin{array}{c}
n \\
{\left[n^{1 / 2}\right]}
\end{array}\right)+\frac{n}{2}
\end{aligned}
$$

for sufficiently large $n$.

From the definition of the binomial coefficients it is immediate that

$$
\log \left(\begin{array}{c}
n \\
{\left[n^{\alpha}\right]}
\end{array}\right)<n^{\alpha} \log n \text { for } 0<\alpha<1
$$

hence,

$$
\begin{aligned}
\log \left(\begin{array}{c}
n \\
{\left[n^{1 / 2}\right]}
\end{array}\right) & <n^{1 / 2} \log n, \\
\log \left(B_{n+1} / P_{n}\right) & <n / 2+n^{1 / 2} \log n
\end{aligned}
$$

for sufficiently large $n$, and Lemma 3 is proved.

Let $V$ be a set with $k+1$ elements. Let $D(V)$ denote the set of diagrams that can be defined with $V$ as vertices and with a minimal vertex $v$ covered by a set $Q$ of $\left[k^{1 / 2}\right]$ vertices, with $|C(Q)| \leqq k / 2$. Set $D_{k+1}=|D(V)|$.

Lемма $4 . \log \left(D_{n+1} / P_{n-\left[n^{1 / 2}\right]}\right) \leqq n^{3 / 2} / 2+4 n$ for $n$ sufficiently large.

Proof. Let $|V|=n+1$. We can obtain all diagrams in $D(V)$ as follows: First, we choose a diagram $H$ on $n-\left[n^{1 / 2}\right]$ of the vertices of $V$. Second, from the remaining $\left[n^{1 / 2}\right]+1$ vertices we choose a vertex $v$ to be minimal. Let $Q$ be the other $\left[n^{1 / 2}\right]$ vertices. Third, we adjoin $v$ and $Q$ to $H$ with all vertices of $Q$ covering $v$, and connect $Q$ to at most $n / 2$ vertices of $H$ in such a way that neither forbidden configuration occurs. Finally we let $v$ be covered by some of the vertices not in $C(Q)$. 
There are $\left(\left[n^{n+1}\right]_{+1}^{n+1}\right)$ subsets of the vertices which can be chosen for $H$, and for each such set there are $P_{n-\left[n^{1 / 2}\right]}$ ways to define a diagram on it. From the $\left[n^{1 / 2}\right]+1$ remaining vertices we can choose $v$ in $\left[n^{1 / 2}\right]+1$ ways. In each diagram in $D(V)$ any vertex in $C(Q)$ must either cover some vertices in $Q$ or be covered by some vertices of $Q$, but not both. For if $a, b \in Q, u \in C(Q)$, and $u$ covers $a$, and $b$ covers $u$, then $u, a, b, v$ form a forbidden quadrilateral. Thus there are $2^{\left[n^{1 / 2}\right]+1}$ possible ways to connect the edges from each vertex in $C(Q)$ to $Q$. There are at most $n / 2$ vertices in $C(Q)$ for which this has to be done, and so there are at most $\left(2^{\left[n^{1 / 2}\right]+1}\right)^{n / 2}$ ways to connect vertices in $C(Q)$ once $C(Q)$ has been chosen. There are at most

$$
\sum_{i=0}^{n / 2}\left(\begin{array}{c}
n-\left[n^{1 / 2}\right] \\
i
\end{array}\right) \leqq \frac{n}{2}\left(\begin{array}{c}
n-\left[n^{1 / 2}\right] \\
\frac{n-\left[n^{1 / 2}\right]}{2}
\end{array}\right)
$$

ways to choose $C(Q)$ once $Q$ has been chosen. Finally, $v$ can be connected in fewer than $2^{n}$ ways to vertices not in $C(Q)$. We have

$$
\begin{aligned}
& \log \left(\frac{D_{n+1}}{P_{n-\left[n^{1 / 2}\right]}}\right) \\
& <\log \left(\begin{array}{c}
n+1 \\
{\left[n^{1 / 2}\right]+1}
\end{array}\right)+\log \left(\left[n^{1 / 2}\right]+1\right)+\frac{n}{2}\left(\left[n^{1 / 2}\right]+1\right) \\
& \quad+\log \frac{n}{2}+\log \left(\begin{array}{l}
n-\left[n^{1 / 2}\right] \\
\frac{n-\left[n^{1 / 2}\right]}{2}
\end{array}\right)+n .
\end{aligned}
$$

Comparing terms of (10), below, with (9), we get

$$
\log \left(\frac{D_{n+1}}{P_{n-\left[n^{1 / 2}\right]}}\right)<n+1+\log n+\frac{n^{3 / 2}}{2}
$$

$$
+\frac{n}{2}+\log n+n+n<\frac{n^{3 / 2}}{2}+4 n \text {. }
$$

Lemma 4 is proved.

The three cases considered in these lemmas include all possible diagrams on $n+1$ vertices for sufficiently large $n$. This is because a diagram either has a vertex adjacent to at most $n / 64$ others $\left(A_{n+1}\right)$, or it does not. If a minimal vertex $v$ is connected to more than $n / 64$ vertices, then it is connected to some set $Q$ containing $\left[n^{1 / 2}\right]$ vertices, for $n$ sufficiently large. Then $C(Q)$ has either at least $n / 2$ vertices 
$\left(B_{n+1}\right)$, or at most $n / 2$ vertices $\left(D_{n+1}\right)$. Hence, we have

$$
P_{n+1} \leqq A_{n+1}+B_{n+1}+D_{n+1} \text {. }
$$

We now proceed to prove the theorem.

Proof of Theorem 1. Let $n>4$ be sufficiently large to satisfy the three preceding lemmas. Then we can choose some number $C>2$ such that for all $m \leqq n$ we have

$$
\log P_{m} \leqq m^{2} / 4+C m^{3 / 2} \log m .
$$

Now we complete the induction by using the lemmas to establish (12) for $n+1$. From Lemma 2 we have

$$
\begin{aligned}
\log A_{n+1} & \leqq \frac{n}{5}+\log P_{n} \leqq \frac{n^{2}}{4}+\frac{n}{5}+C n^{3 / 2} \log n \\
& <\left(\frac{n+3}{4}\right)^{2}+C(n+1)^{3 / 2} \log (n+1)-2 .
\end{aligned}
$$

From Lemma 3 we have

$$
\begin{aligned}
\log B_{n+1} \leqq & \log P_{n}+\frac{n}{2}+n^{1 / 2} \log n \\
\leqq & \frac{n^{2}}{4}+C n^{3 / 2} \log n+\frac{n}{2}+n^{1 / 2} \log n \\
< & \frac{(n+1)^{2}}{4}-\frac{n}{2}-\frac{1}{4}+C\left(n^{3 / 2}+\frac{3}{2} n^{1 / 2}\right) \log n \\
& -\frac{3}{2} C n^{1 / 2} \log n+\frac{n}{2} \log n \\
< & \frac{(n+1)^{2}}{4}+C(n+1)^{3 / 2} \log (n+1)-n^{1 / 2} \log n .
\end{aligned}
$$

Finally from Lemma 4 we have

$$
\begin{aligned}
\log D_{n+1} & \leqq \log P_{n-[n 1 / 2]}+\frac{n^{3 / 2}}{2}+4 n \\
& \leqq \frac{\left(n-\left[n^{1 / 2}\right]\right)^{2}}{4}+C\left(n-\left[n^{1 / 2}\right]\right)^{3 / 2} \log \left(n-\left[n^{1 / 2}\right]\right)+\frac{n^{3 / 2}}{2}+4 n \\
& <\frac{n^{2}}{4}+\frac{n}{4}+C n^{3 / 2} \log n-\frac{3}{2} C n \log n+4 n \\
& <\frac{(n+1)^{2}}{4}+C(n+1)^{3 / 2} \log (n+1)-n \log n .
\end{aligned}
$$


Let $N=(n+1)^{2} / 4+C(n+1)^{3 / 2} \log (n+1)$. Then (13), (14), (15) imply

$$
A_{n+1}<2^{N} \cdot \frac{1}{4}, \quad B_{n+1}<2^{N} \cdot \frac{1}{4}, \quad D_{n+1}<2^{N} \cdot \frac{1}{4} .
$$

Adding these upper bounds for $A_{n+1}, B_{n+1}$, and $D_{n+1}$ and using (11) establishes (12) for $n+1$ and completes the induction. This proves Theorem 1.

It is interesting to note that the constant $C$ in Theorem 1 plays no role in the proofs of Lemmas 2 to 4 . It serves only to begin the induction in the proof of Theorem 1.

\section{REFERENCES}

1. G. Birkhoff, Lattice theory, Amer. Math. Soc. Colloq. Publ., vol. 25, Amer. Math. Soc., Providence, R. I., 1940; 3rd ed., 1967. MR 1, 325; MR 37 \#2638.

2. S. D. Chatterji, The number of topologies on $n$ points, Kent State University, NASA Technical Report, 1966.

3. L. Comtet, Recouvrements, bases de filtre et topologies d'un ensemble fini, C. R. Acad. Sci. Paris. Sér. A-B 262 (1966), A1091-A1094. MR 34 \#1209.

4. J. W. Evans, F. Harary and M. S. Lynn, On the computer enumeration of finite topologies, Comm. ACM 10 (1967), 295-298. 1136.

5. J. Kelley, General topology, Van Nostrand, Princeton, N. J., 1955. MR 16,

6. D. Klarner, The number of graded partially ordered sets, J. Combinatorial Theory 6 (1969), 12-19. MR 38 \#4333.

7. - The number of classes of isomorphic graded partially ordered sets, (to appear).

8. V. Krishnamurthy, On the number of topologies on a finite set, Amer. Math. Monthly 73 (1966), 154-157. MR $34 \# 1208$.

9. O. Ore, Theory of graphs, Amer. Math. Soc. Colloq. Publ., vol. 38, Amer. Math. Soc., Providence, R. I., 1962. MR 27 \#740.

Massachusetts Institute of Technology, Cambridge, Massachusetts 02139 and University of California, Los Angeles, California 90024 